\title{
EFECTIVIDAD DE LA BIODANZA® EN PERSONAS CON TRASTORNO MENTAL GRAVE Y FAMILIARES EN UN HOSPITAL DE DÍA
}

BIOdANZA® EFFECTIVENESS IN PEOPLE WITH SEVERE MENTAL DISORDER AND FAMILY IN A DAY HOSPITAL

Ma Mar Alguacil Cabrera, David Lozano Díaz, Ángel Arias Arias

Hospital General La Mancha Centro, Alcázar de San Juan, Ciudad Real, España.

Alguacil Cabrera, M., Lozano Díaz, D., \& Árias Árias, Á. (2020). EFECTIVIDAD DE LA BIODANZA EN PERSONAS CON TRASTORNO MENTAL GRAVE Y FAMILIARES EN UN HOSPITAL DE DÍA. Revista Ene De Enfermería, 14(1). Consultado de http://www.eneenfermeria.org/ojs/index.php/ENE/article/view/940 
Alguacil-CABrera - LOZANo-Díaz - ARIAS-ARIAS Biodanza en personas con trastorno Mental Grave y familiares en un Hospital de Día

\section{Resumen}

Objetivo: Evaluar la efectividad de la intervención con Biodanza® de tres meses en personas con Trastorno mental grave (TMG) y familiares. Analizar los cambios en calidad de vida (SF-36), niveles de ansiedad-depresión (HAD) y autoestima (RSE) provocados por la intervención.

Material y Métodos: Estudio preexperimental, pretest-postest sin grupo control. Se realizó en el Hospital de Día de la Unidad de Salud Mental del Hospital General Mancha Centro. Participaron 21 personas, once con trastorno mental grave y diez familiares. La intervención Biodanza® se llevó a cabo por una enfermera una vez por semana durante tres meses en pacientes y familiares a la vez, como complemento al tratamiento estándar. Se recogieron datos sociodemográficos, se midió la calidad de vida (SF-36), ansiedad-depresión (HAD) y autoestima (RSE) antes y después de la intervención.

Resultados: La edad media fue de $46,4(14,2)$ años, el 80,9\% eran mujeres. En cuanto a la calidad de vida, se observaron diferencias significativas (función física $(58,5 \pm 30,6$ vs $77,3 \pm 19,5)$; $p=0,001)$, salud general $(38,4 \pm 16,1$ vs $55,3 \pm 22,3 ; p=0,001)$, vitalidad $(38,2 \pm 17,8$ vs $55,6 \pm 18,7 ; p=0,001)$, función social $(40,4 \pm 22,8$ vs $68,4 \pm 23,8 ; p=0,003)$ y sa- lud mental $(39,1 \pm 20,7$ vs $56,2 \pm 15,7$; $p=0,01)$. Se observaron diferencias significativas en los niveles de ansiedad $(13,3 \pm 3,9$ vs $7,5 \pm 3,4 ; p<0,001)$, y en niveles de depresión $(11,3 \pm 5$ vs $5,9 \pm 3,4$; $p=0,001)$. La autoestima mejoró significativamente $(24,6 \pm 5,7$ vs $28,5 \pm 4,7$; $p=0,009$ ), al comparar pre-test y postest.

Conclusiones: La intervención con Biodanza ${ }^{\circledR}$ junto al tratamiento estándar mejora la calidad de vida, autoestima y disminuye la ansiedad y depresión en personas con trastorno mental grave y familiares a los tres meses (con una sesión por semana).

\section{Palabras Clave}

Biodanza, Trastorno Mental Grave, Ansiedad, Depresión, Autoestima, Calidad de Vida. 
Abstract

Objective: To evaluate the effectiveness of Biodanza ${ }^{\circledR}$ intervention during three months in people with severe mental disorder and their family members. Analyze changes in quality of life (SF-36), levels of anxiety-depression (HAD) and self-esteem (RSE) caused by the intervention.

Material and Methods: A pre-experimental study, pretest-posttest without a control group. It was held at the Day Hospital of the Mental Health Unit of the General Hospital Mancha Centro. They included twentyone people, eleven with severe mental disorder and ten family members. The Biodanza ${ }^{\circledR}$ intervention took place by a nurse once a week for three months in patients and family at a time, in addition to standard treatment. Socio-demographic data were collected, the quality of life (SF-36), anxiety-depression (HAD) and self-esteem (RSE) were measured before and after the intervention.

Results: The mean age was 46.4 (14.2) years, $80.9 \%$ were women. As for quality of life, significant differences were observed (physical function (58.5 \pm 30.6 vs $77.3 \pm 19.5 ; p=0.001$ ), general health $(38.4 \pm 16.1$ vs $55.3 \pm 22.3 ; p=0.001)$, vitality $(38.2 \pm 17.8$ vs $55.6 \pm 18.7 ; p=$ $0.001)$, social function $(40.4 \pm 22.8$ vs $68.4 \pm 23.8 ; p=0.003$ ) and mental health
(39.1 \pm 20.7 vs. $56.2 \pm 15.7 ; p=0.01)$. Significant differences were observed in anxiety levels $(13.3 \pm 3.9$ vs $7.5 \pm 3.4 ; p$ $<0.001)$, and in levels of depression (11.3 \pm 5 vs $5.9 \pm 3.4 ; p=0.001)$. Self-esteem improved significantly (24.6 \pm 5.7 vs 28.5 \pm 4.7 ; $p=0.009$ ), when comparing pretest and post-test.

Conclusions: The Biodanza ${ }^{\circledR}$ intervention, when aplied together with standard treatment improves quality of life, self-esteem and reduces anxiety and depression in people with severe mental disorder and their family members after three months (with one session per week).

\section{Keywords}

Biodanza, Severe mental disorder, Quality of life, Anxiety, Depression. 
ALgUACIL-CABRERA - LOZANO-DíaZ - ARIAS-ARIAS BIODANZA EN PERSONAS CON TRASTORNO MENTAL GRAVE y FAMILIARES EN un HOSPital de Día

\section{INTRODUCCIÓN}

Los trastornos mentales son un problema de primera magnitud, por su elevada prevalencia (estimaciones del 15 y $25 \%$ de la población general los padece en algún momento de sus vidas) y por el impacto de sufrimiento y desestructuración de las personas, sus familias y su entorno más cercano (1).

El concepto de Trastorno Mental Grave (TMG) utilizado habitualmente es el que formuló el Instituto Nacional de Salud Mental de Estados Unidos (NIMH) en 1987 donde incluye tres dimensiones (diagnóstico de trastornos psicóticos y algunos trastornos de personalidad; duración de la enfermedad y del tratamiento superior a los dos años; y presencia de discapacidad o afectación moderada o severa del funcionamiento laboral, social y familiar) y engloba diversos trastornos mentales.

En los últimos años se han aportado numerosas novedades terapéuticas en el ámbito de lo farmacológico (nuevos antipsicóticos atípicos o de segunda generación, nuevos estabilizadores del humor, etc.), que han contribuido a mejorar la evolución de las personas con TMG. El control parcial y limitado de la sintomatología, los efectos secundarios a corto y largo plazo, y la pobre adherencia al tratamiento de un porcentaje no desdeñable de personas afectadas, plantean la necesidad de utilizar un enfoque más amplio, en el que el tratamiento farmacológico se complemente con otras intervenciones psicoterapéuticas y psicosociales. Se han de coordinar y aplicar de manera eficiente para conseguir la recuperación y la mejora de aspectos relacionados con el funcionamiento en distintas áreas de la conducta, de la integración social, laboral y familiar, generando un considerable consumo de recursos sociosanitarios y ocasionando un importante impacto económico (2).

Por tanto, el tratamiento integral constituye uno de los principales desafíos de los dispositivos de atención a la salud mental. El objetivo final de la atención integral a la persona con TMG es, como han señalado diversos autores (3-5), lograr que la persona recupere su proyecto vital tras la aparición de la enfermedad y la discapacidad. No solo implica que desaparezcan los síntomas, o disminuyan las discapacidades, sino que supone apoyarse en las fortalezas, en las potencialidades que quedan y en el proceso dinámico de querer seguir desarrollándose en esa nueva situación.

Por otro lado, existe un consenso general en cuanto a que el TMG afecta de manera considerable las relaciones en las familias de las personas que lo sufren, y que dichas relaciones también pueden afectar de algún modo el curso 
ALGUACIL-CABRERA - LOZANO-DÍAZ - ARIAS-ARIAS BIODANZA EN PERSONAS CON TRASTORNo MENTAL GRAVE y FAMILIARES EN un HOSPITAL DE Día

de la enfermedad. Hay evidencia que muestra que los pacientes que han recibido intervención familiar tienen mayor nivel de calidad de vida (6). En las recomendaciones de la Guía de Práctica Clínica (GPC) de Intervenciones Psicosociales en el TMG encontramos que a las personas con TMG, diagnóstico esquizofrénico y relacionados, y a sus familias, se les podrá ofrecer otras intervenciones terapéuticas como complemento terapéutico del tratamiento estándar. Investigaciones recientes permiten recomendar la aplicación de técnicas expresivas, con independencia de la modalidad utilizada (arteterapia, musicoterapia, etc.) realizada por profesionales con formación especializada acreditada en pacientes con sintomatología negativa $(7,8)$.

El contexto de Hospital de Día (HD) puede ser una valiosa oportunidad para poner en marcha programas e intervenciones que atiendan y den respuesta a las personas con TMG, así como a sus familiares.

Biodanza ${ }^{\circledR}$ es un método terapéutico que utiliza la interacción con la música, el movimiento y la emoción como medio de comunicación y expresión. Se define como un sistema vivencial de integración humana, renovación orgánica, reeducación afectiva, y reaprendizaje de las funciones originarias de vida. Su metodología consiste en inducir vivencias integradoras, que estimulan la expresión de los potenciales humanos (9). Actúa prioritariamente en el sistema integrador-adaptativo límbicohipotalámico y esto contribuye a la consolidación de modelos de comportamiento e influye sobre la corteza cerebral a través de sus conexiones neocorticales, generando estados de armonía e integración y favoreciendo aumentos muy significativos en los índices de vitalidad, equilibrio emocional, disminución del estrés, fortalecimiento de la identidad y la autoestima (10).

Por sus características terapéuticas, Biodanza ${ }^{\circledR}$ representa una importante posibilidad para dar cobertura a las necesidades humanas y afectivas de los pacientes con TMG y sus familiares en el contexto del HD. Estudios recientes han demostrado la eficacia de Biodanza ${ }^{\circledR}$ para pacientes con fibromialgia, produciendo un aumento en la calidad de vida y cambios beneficiosos sobre el dolor y la motricidad (11-14). López-Rodríguez et al. demostraron que Biodanza $\AA^{\circledR}$ tiene un efecto positivo sobre el estrés percibido y la depresión en adultos jóvenes(15). También existe una revisión con relevancia de ensayos controlados aleatorizados (ECA) con Musicoterapia para pacientes con esquizofrenia y trastornos similares, donde se demostró la eficacia de las técnicas expresivas en TMG (16). Por la 
Alguacil-CABrera - LOZANo-Díaz - ARIAS-ARIAS BIODANZA EN PERSONAS CON TRASTORNo MENTAL GRAVE y FAMILIARES EN un HOSPITAL DE Día

similitud terapéutica entre Biodanza ${ }^{\circledR}$ y

Musicoterapia en cuanto a que los dos son métodos terapéuticos que utilizan la interacción musical como un medio de comunicación y expresión, hemos considerado que Biodanza ${ }^{\circledR}$ podría facilitar una mejora en la calidad de vida de personas con TMG.

Considerando las recomendaciones de la GPC, teniendo en cuenta el contexto favorable que ofrece el dispositivo HD y que en la actualidad no existen estudios publicados que valoren la efectividad de Biodanza ${ }^{\circledR}$ en personas con TMG y en sus familiares se ha planteado esta intervención. El objetivo de este estudio es analizar la efectividad de Biodanza ${ }^{\circledR}$ agregada en un grupo de personas con TMG y familiares en el HD del Hospital General La Mancha Centro, evaluando la calidad de vida, los niveles de ansiedad-depresión y la autoestima, de estas personas pre-test y pos-test; y evaluar el efecto diferencial entre personas con TMG y sus familiares al recibir la intervención.

\section{MATERIAL Y MÉTODO}

\section{Diseño y población de estudio}

Se realizó un estudio pre-experimental, pretest-postest sin grupo control en el marco de atención de HD, formado por un grupo de intervención (Biodanza ${ }^{\circledR}$ en pacientes con TMG y sus familiares).
Los criterios de inclusión fueron: personas con TMG diagnosticado y en tratamiento estándar en HD del servicio de Salud Mental del HGLMC y los familiares de las personas con TMG que les acompañan y/o asisten al grupo multifamiliar del HD; personas comprendidas entre los 16 y 75 años de edad; y que expresaron su voluntad de participar mediante la firma de un consentimiento informado, fueron reclutados por el equipo de profesionales de salud mental de HD (2 psiquiatras y 1 psicóloga clínica).

Por último, se excluyeron de los análisis finales aquellos sujetos que no participaron en al menos 7 sesiones de terapia.

\section{Intervención}

La metodología de Biodanza ${ }^{\circledR}$ fue realizada por una enfermera, especializada en Biodanza Clínica (Sistema Rolando Toro, formación de 3 años). Dicha metodología se hizo a través de músicas y ejercicios específicos que inducían vivencias integradoras en los participantes, permitiéndoles identificar en sí mismos herramientas para una mejor utilización de sus potencialidades internas, para desarrollar su afectividad y creatividad y para ampliar la capacidad de tomar conciencia.

Para ello se realizó un programa de 12 sesiones de Biodanza ${ }^{\circledR}$, teniendo 
Alguacil-CABrera - LOZANo-Díaz - ARIAS-ARIAS BIODANZA EN PERSONAS CON TRASTORNO MENTAL GRAVE Y FAMILIARES EN un HOSPItAL DE Día

en cuenta las necesidades personales percibidas y recogidas mediante un cuestionario antes del inicio de las sesiones, por cada uno de los participantes, para ofrecer una atención asistencial individualizada y centrada en los mismos. El tiempo de duración de cada sesión fue de dos horas. Dichas sesiones se realizaron una vez por semana, durante un periodo de 3 meses. Los pacientes y familiares continuaron simultáneamente con sus terapias habituales del HD. Cada sesión de Biodanza ${ }^{\circledR}$ constaba de dos partes impartidas de forma consecutiva.

Primera parte: "Relato de vivencia", con una duración de 30 minutos. Parte de comunicación verbal donde cada participante expresa sus ideas y vivencias.

Segunda parte: "Vivencial", con una duración de 1:30 horas. Parte no verbal y práctica, con música, ejercicios y encuentros afectivos tanto individuales como en grupo.

La temática, las músicas, las consignas y los ejercicios planteados se diseñan para cada sesión específica y centrados en las necesidades percibidas en el grupo.

\section{Variables e instrumentos}

Con el propósito de evaluar los cambios que la intervención provoca en los participantes se utilizaron los siguientes instrumentos:

Cuestionario de Calidad de Vida - SF-36 (17) - (versión española). Evalúa la calidad de vida relacionada con la salud, siendo un marcador indirecto del estado de salud global y de adaptación social de la persona y por lo tanto del impacto de la intervención (18). Consta de 36 ítems autoaplicados y nos mide el momento actual en 8 dimensiones: Función física (FF), rol físico (RF), dolor corporal (DC), salud general (SG), vitalidad $(V T)$, función social (FS), rol emocional (RE) y salud mental (SM).

Escala de Ansiedad y Depresión de Goldberg - HAD (19) - (versión española). Evalúa los niveles de ansiedad y depresión con 14 ítems autoaplicados. Se trata de un test que orienta el diagnóstico hacia ansiedad o depresión (o ambas en casos mixtos), discrimina entre ellos y dimensiona sus respectivas intensidades.

Escala de Autoestima de Rosenberg - RSE (20)- (versión española). Evalúa el grado de autoestima con 10 ítems autoaplicados.

Los instrumentos SF-36, HAD y RSE fueron administrados antes del inicio y al final de la intervención con Biodanza ${ }^{\circledR}$ por la investigadora.

Se recogieron las principales variables sociodemográficas como: edad, 
ALgUACIL-CABRERA - LOZANo-DíaZ - ARIAS-ARIAS BIODANZA en personas con TRAstorno Mental Grave y FAmiliares en un Hospital de DÍa

sexo, paciente o familiar, diagnóstico psiquiátrico $\mathrm{CIE}-10$, tratamiento farmacológico, situación laboral, $\mathrm{n}^{\circ}$ de sesiones recibidas y necesidades personales percibidas.

\section{Análisis estadístico}

Se ha realizado un análisis descriptivo de todas las variables incluidas en el estudio. Las variables cualitativas se han expresado como frecuencias absolutas y relativas y las variables cuantitativas como medidas de tendencia central (media o mediana) y medidas de dispersión (desviación estándar o rango intercuartílico) según su distribución. Se ha utilizado el test de Wilcoxon para datos apareados en el análisis de los resultados de los cuestionarios antes y después de las sesiones de Biodanza ${ }^{\circledR}$. Se mantuvo un nivel de confianza de 95\% $(a=0,05)$. Las correlaciones entre los distintos cuestionarios se han realizado mediante la correlación de Spearman. Se considerará como estadísticamente significativo un valor de $p<0,05$. Para el análisis estadístico se ha utilizado el programa informático PASW v18 (SPSS Inc.).

\section{Aspectos éticos}

El proyecto fue aprobado por el Comité Ético de Investigación Clínica del Hospital General La Mancha Centro. Todos los participantes firmaron un consentimiento informado y se siguieron los criterios éticos de la declaración de Helsinki.

\section{Resultados}

Un total de 21 sujetos (11 pacientes y 10 familiares), de un total de 18 pacientes y 54 familiares aceptaron participar y firmaron el consentimiento informado (Figura 1). De ellos, el 80,9 \% mujeres $(n=17)$ y el $19,1 \%$ hombres $(n=4)$, con una edad media de 46,4 4 14,2 años. Los 11 pacientes (8 fueron mujeres y 3 hombres) tenían una edad media de $38,9 \pm 11$ años, mientras que los 10 familiares ( 9 fueron mujeres y 1 hombre) tenían una edad media de 54,6 \pm 13 años. 


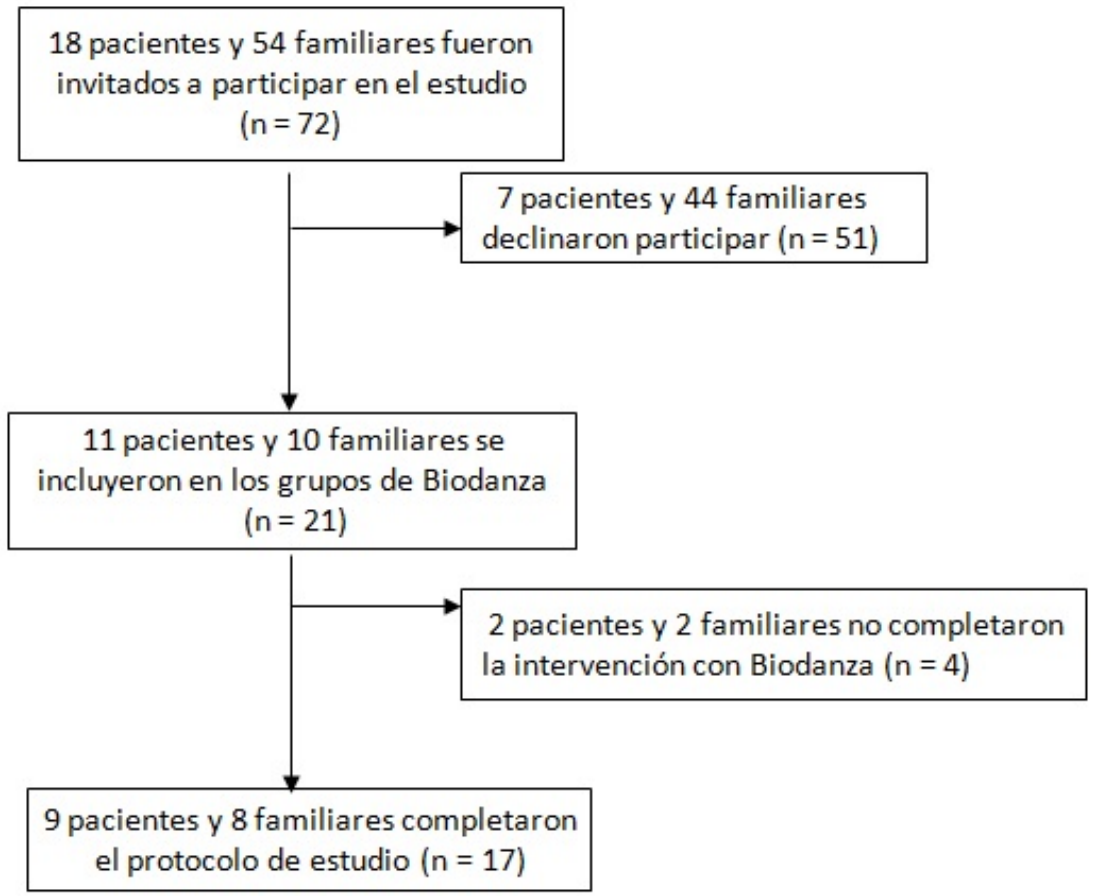

Figura 1. Diagrama de flujo de los participantes en el estudio.

El $71 \%$ de los participantes asistió a 9 o más sesiones. La media de sesiones a las que asistieron los participantes fue 9,5 sesiones. Durante las sesiones hubo 4 abandonos (2 familiares y 2 pacientes) que dejaron de acudir voluntariamente, por lo que 17 participantes llegaron al final del proceso (81\%). De ellos 9 fueron pacientes y 8 familiares. La motivación para la intervención fue alta y se mantuvo estable a lo largo de los tres meses que duró el estudio.

Los pacientes tenían los siguientes diagnósticos psiquiátricos según la clasificación CIE-10: trastorno de personalidad $(n=5)$, trastorno límite de personalidad $(n=1)$, depresión mayor $(n=1)$, trastorno disociativo $(n=1)$, trastorno psi- cótico $(n=1)$. Adicionalmente, un familiar refirió ansiedad y otro depresión. Todos los pacientes tenían tratamiento farmacológico estándar.

En cuanto a la situación laboral de los participantes fue distinta en pacientes y familiares; el $64 \%$ de los pacientes estaban desempleados, el $27 \%$ tenía declarada incapacidad y el $9 \%$ estaba de baja laboral. De los familiares, el $40 \%$ estaba activo, el $30 \%$ trabajaba en sus labores, el $20 \%$ desempleado, y el $10 \%$ estaba jubilado. 


\section{Calidad de Vida: SF-36}

Los pacientes tenían peor puntuación inicial que los familiares en las 8 dimensiones del cuestionario SF-36. Después de la intervención con Biodan$\mathrm{za}{ }^{\circledR}$ todas las dimensiones mejoraron tanto en los pacientes como en los familiares, se observaron diferencias significativas en la puntuación de las dimensiones de funcionalidad física (FF), salud general (SG), vitalidad (VT), función social (FS) y salud mental (SM), estas dimensiones que mejoraron significativamente fueron iguales en pacientes y familiares, salvo la SM que mejoró significativamente en los pacientes pero no en los familiares $(p=0,042$ y $p=0,091$, respectivamente). No se observan diferencias significativas en el rol físico (RF), rol emocional (RE) y dolor corporal (DC) (Tabla 1). 


\begin{tabular}{|c|c|c|c|}
\hline Dimensiones Cuestionario SF36 & Basal & Final & $\mathrm{p}$ \\
\hline \multicolumn{4}{|l|}{ Global } \\
\hline Funcionalidad Física (FF) & $58.5(30.6)$ & $77.4(19.5)$ & .001 \\
\hline Rol Físico (RF) & $52.9(44.1)$ & $67.6(39.3)$ & .111 \\
\hline Dolor Corporal (DC) & $50.7(30.4)$ & $64.4(32.8)$ & .077 \\
\hline Salud General (SG) & $38.4(16.1)$ & $55.3(22.3)$ & .001 \\
\hline Vitalidad (VT) & $38.2(17.8)$ & $55.6(18.7)$ & .001 \\
\hline Función Social (FS) & $40.4(22.8)$ & $68.4(23.8)$ & .003 \\
\hline Rol Emocional (RE) & $21.6(28.7)$ & $39.6(45.9)$ & .188 \\
\hline Salud Mental (SM) & $39.1(20.7)$ & $56.2(15.7)$ & .010 \\
\hline \multicolumn{4}{|l|}{ Pacientes } \\
\hline Funcionalidad Física (FF) & $53.3(35.7)$ & $68.3(19.4)$ & .041 \\
\hline Rol Físico (RF) & $25(39.5)$ & $44.4(39.1)$ & .161 \\
\hline Dolor Corporal (DC) & $39.4(32.7)$ & $53.3(29.8)$ & .205 \\
\hline Salud General (SG) & $25(6.5)$ & $43.9(19.2)$ & .017 \\
\hline Vitalidad (VT) & $30.6(11.3)$ & $48.9(16.2)$ & .007 \\
\hline Función Social (FS) & $29.2(18.8)$ & $58.3(25)$ & .024 \\
\hline Rol Emocional (RE) & $11.1(16.7)$ & $25.9(43.4)$ & .336 \\
\hline Salud Mental (SM) & $34.7(20.1)$ & $52.9(17.8)$ & .042 \\
\hline \multicolumn{4}{|l|}{ Familiares } \\
\hline Funcionalidad Física (FF) & $64.4(24.7)$ & $87.5(14.9)$ & .011 \\
\hline Rol Físico (RF) & $84.4(22.9)$ & $93.8(17.7)$ & .450 \\
\hline Dolor Corporal (DC) & $63.4(23.2)$ & 76.9 (33.3) & .203 \\
\hline Salud General (SG) & $51.9(10)$ & $68.1(19.1)$ & .021 \\
\hline Vitalidad (VT) & $46.9(20.5)$ & $63.1(19.4)$ & .028 \\
\hline Función Social (FS) & $53.1(20.9)$ & $79.7(17.6)$ & .048 \\
\hline Rol Emocional (RE) & $33.3(35.6)$ & $57.1(46)$ & .395 \\
\hline Salud Mental (SM) & $44(21.6)$ & $60(13.2)$ & .091 \\
\hline
\end{tabular}

Tabla 1. Puntuaciones en el cuestionario SF-36 (calidad de vida) en los pacientes y familiares antes y después de la intervención con Biodanza

\section{Ansiedad-depresión: HAD}

Respecto a la escala de ansiedad y depresión de Goldberg (HAD), los pacientes también partían de una peor si- tuación basal en cuanto a depresión y ansiedad que los familiares. Se detecta un descenso estadísticamente significativo tanto en la ansiedad de los partici- 
pantes tras la intervención con Biodan-

$z a \circledR(13,3 \pm 3,9$ vs $7,5 \pm 3,4 ; p<0,001)$

como en la depresión (11,3 \pm 5 vs $5,9 \pm$

$3,4 ; p=0,001)$. Analizando por subgrupos, observamos que tanto en los pacientes como en los familiares se produce una disminución estadísticamente significativa en su ansiedad ( $p=0,011$ y $p=0,012$, respectivamente) y en la depresión ( $p=0,017$ y $p=0,018$, respectivamente) tras la intervención con Bio$\operatorname{danza}{ }^{\circledR}($ Tabla 2$)$.

\begin{tabular}{lccc}
\hline \multicolumn{1}{c}{ Cuestionario HAD } & Basal & Final & $p$ \\
\hline Ansiedad & & & \\
Global & $13,3(3,9)$ & $7,5(3,4)$ & $<0,001$ \\
Pacientes & $14(3,6)$ & $8,7(3,9)$ & 0,011 \\
Familiares & $12,5(4,3)$ & $6,3(2,5)$ & 0,012 \\
Depresión & & & \\
Global & $11,3(5)$ & $5,9(3,4)$ & 0,001 \\
Pacientes & $12,6(5,2)$ & $7,6(2,9)$ & 0,017 \\
Familiares & $9,9(4,8)$ & $4(3)$ & 0,018 \\
\hline
\end{tabular}

Tabla 2. Puntuaciones en el cuestionario HAD (ansiedad - depresión) en los pacientes y familiares antes y después de la intervención con Biodanza

\section{Autoestima: RSE}

Los pacientes tienen una menor autoestima basal que los familiares medida por la escala de Rosenberg (RSE). Se observa una mejora estadísticamente significativa en la autoestima de los participantes tras la intervención con Biodanza ${ }^{\circledR}(24,6 \pm 5,7$ vs $28,5 \pm 4,7 ; p=$ $0,009)$, pasando de autoestima baja (< 25 puntos) a autoestima media (26-29 puntos). Si analizamos por subgrupos, se aprecia una mejora estadísticamente significativa en la autoestima de los pacientes $(p=0,049)$ pero no alcanza la significación en los familiares $(p=0,122)$ tras la intervención con Biodanza ${ }^{\circledR}$ (Tabla 3). 


\begin{tabular}{cccc}
\hline Cuestionario RSE & Basal & Final & $\mathrm{p}$ \\
\hline Global & $24,6(5,7)$ & $28,5(4,7)$ & 0,009 \\
Pacientes & $23,1(5,6)$ & $27,3(6)$ & 0,049 \\
Familiares & $26,4(5,7)$ & $29,9(2,5)$ & 0,122 \\
\hline
\end{tabular}

Tabla 3. Puntuaciones en el RSE (autoestima) en los pacientes y familiares antes y después de la intervención con Biodanza.

\section{Correlación entre escalas}

Se analizaron las correlaciones entre las escalas utilizadas, observándose una correlación negativa y estadísticamente significativa entre la ansiedad y
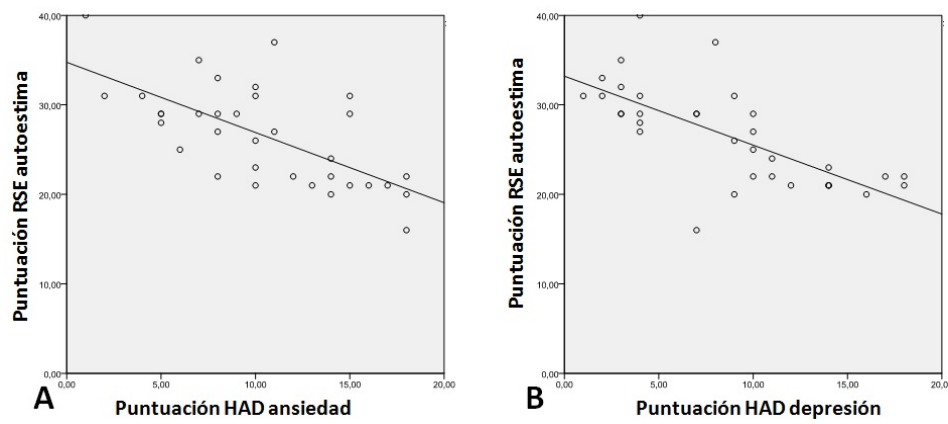

entre la depresión y autoestima (rho = $-0,751 ; p<0,001)$, sin embargo se observó una correlación positiva y significativa entre depresión y ansiedad (rho = 0,692; $\mathrm{p}<0,001$ ) (Figura 2).

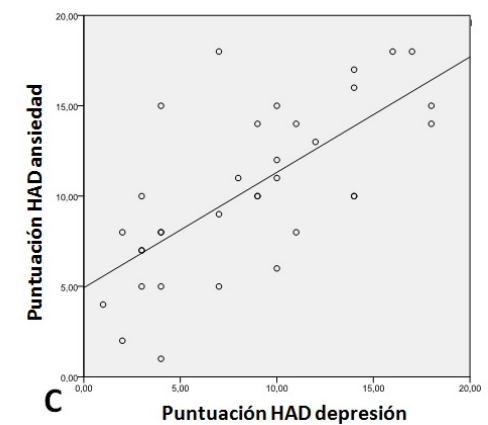

la autoestima (rho $=-0,633 ; p<0,001$ ) y

Figura 2. Correlaciones entre las escalas de autoestima, ansiedad y depresión

Todas las dimensiones del cuestionario SF-36 se correlacionaron positiva y significativamente entre sí y con la autoestima y significativamente de forma negativa con la ansiedad y la depresión, excepto FF con RSE y DC con depresión, ansiedad y autoestima (Tabla 4). 


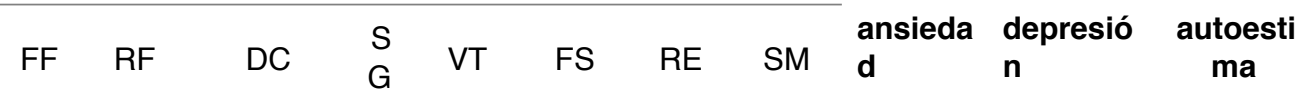

\begin{tabular}{|c|c|c|c|c|c|c|c|c|c|c|c|}
\hline \multicolumn{12}{|l|}{ SF36 } \\
\hline $\mathrm{FF}$ & - & $.570^{\star}$ & $.801^{*}$ & $.542^{*}$ & $.535^{\star}$ & $.484^{*}$ & $.375^{*}$ & $.527^{\star}$ & $-.391^{\star}$ & $350^{*}$ & . 154 \\
\hline RF & - & - & $.635^{\star}$ & $.751^{*}$ & $.448^{*}$ & $.636^{\star}$ & $.615^{\star}$ & $.457^{\star}$ & $-.453^{\star}$ & $384^{*}$ & $.392^{*}$ \\
\hline $\mathrm{DC}$ & - & - & - & $.611^{*}$ & $.541^{*}$ & $.511^{*}$ & $.525^{*}$ & $.417^{\star}$ & -.292 & -.213 & .212 \\
\hline$S G$ & - & - & - & - & $.649^{*}$ & $.698^{*}$ & $.538^{\star}$ & $.587^{\star}$ & $-.510^{\star}$ & $\begin{array}{c}- \\
570^{*}\end{array}$ & $.653^{*}$ \\
\hline VT & - & - & - & - & - & $.734^{*}$ & $.461^{*}$ & $.824^{*}$ & $-.476^{\star}$ & $\begin{array}{c}- \\
711^{*}\end{array}$ & $.489^{\star}$ \\
\hline FS & - & - & - & - & - & - & $.506^{\star}$ & $.717^{\star}$ & $-.642^{\star}$ & $\begin{array}{c}- \\
670^{*}\end{array}$ & $.579^{\star}$ \\
\hline $\mathrm{RE}$ & - & - & - & - & - & - & - & $.500^{\star}$ & $-.392^{\star}$ & $\begin{array}{c}- \\
408^{*}\end{array}$ & $.531^{*}$ \\
\hline SM & - & - & - & - & - & - & - & - & $-.621^{\star}$ & $\begin{array}{c}-. \\
786^{*}\end{array}$ & $.503^{\star}$ \\
\hline $\begin{array}{l}\text { HAD } \\
\text { ansieda } \\
\text { d }\end{array}$ & - & - & - & - & - & - & - & - & - & $692^{*}$ & $-.633^{\star}$ \\
\hline $\begin{array}{l}\text { HAD } \\
\text { depresi } \\
\text { ón }\end{array}$ & - & - & - & - & - & - & - & - & - & - & $-.751^{*}$ \\
\hline $\begin{array}{l}\text { RSE } \\
\text { autoesti } \\
\text { ma }\end{array}$ & - & - & - & - & - & - & - & - & - & - & - \\
\hline
\end{tabular}

Tabla 4. Correlaciones entre las escalas utilizadas en el estudio $\left({ }^{\star} p<.05\right)$

\section{Discusıón \\ Los resultados obtenidos en nuestro estudio permiten afirmar que la Biodanza® puede ser efectiva en perso- nas con TMG y familiares en el ámbito de los Hospitales de Día. Se observaron diferencias significativas en el grupo de participantes (tanto en pacientes como en familiares) tras la realización de la intervención para las variables calidad de}

vida, ansiedad, depresión y autoestima. Analizando por subgrupos, observamos que las mejoras más significativas se han obtenido en los pacientes. En los familiares también se obtienen mejoras en dichas variables, excepto en la autoestima, que aunque mejora, no es significativa.

El TMG persistente tiene un gran impacto en la vida de las personas que lo padecen, por lo que el interés de la 
ALGUACIL-CABRERA - LOZANO-DÍAZ - ARIAS-ARIAS BIODANZA EN PERSONAS CON TRASTORNO MENTAL GRAVE Y FAMILIARES EN un HOSPItAL DE Día

calidad de vida ha sido una constante en el desarrollo de los programas y servicios de atención a este colectivo ${ }^{(21)} \mathrm{y}$, de hecho, su mejora supone a menudo uno de sus objetivos explícitos (22). Por tanto, la calidad de vida es una variable relevante en la valoración de programas de intervención psicosocial, en general, y en los dirigidos a la rehabilitación de las personas con TMG $(23,24)$. En nuestro estudio la calidad de vida medida por el cuestionario SF-36 es la variable que más ha mejorado tras la intervención, con mejoras en los pacientes superiores al $76 \%$ en RE, FS, RF y SG, y superiores al $52 \%$ en VT y SM, y en los familiares mejoras superiores al $50 \%$ en RE y FS, aunque el RE no obtuviera significación estadística en ambos subgrupos.

Castañeda (25), tras su estudio en un grupo de practicantes de Biodanza ${ }^{\circledR}$ en relación con la promoción de la salud, encontró que los participantes entienden la Biodanza ${ }^{\circledR}$ como una práctica que genera bienestar y ayuda a la mejora de la calidad de vida de las personas, ya que con una práctica regular se logra el equilibrio corporal, la integración afectiva y la renovación orgánica, además de disolver tensiones motoras crónicas, favoreciendo el buen humor, hecho que refuerza la autoestima y la valoración del propio cuerpo, estimulando el contacto interpersonal.
Se han realizado estudios previos sobre el efecto de la Biodanza ${ }^{\circledR}$ en la calidad de vida, ansiedad, depresión e inteligencia emocional en distintos grupos, pero hasta la fecha no se han realizado trabajos con pacientes de TMG y sus familiares. Por una parte, Abad Robles et al. (26), encuentran que Biodan$\mathrm{za}{ }^{\circledR}$ mejora la inteligencia emocional percibida en 35 mujeres de mediana edad de un nivel socioeconómico mediobajo tras 10 sesiones. Por otra parte, en pacientes con fibromialgia, López-Rodríguez et al. (13) observan que la Biodanza® acuática mejora la calidad de vida, la depresión y el dolor tras 12 semanas de intervención (2 sesiones de 1 hora/ semana) versus un grupo control de estiramientos. En una investigación posterior del mismo grupo, encuentran que dicha intervención mejora la calidad de vida, la ansiedad y la calidad del sueño en estos pacientes (27).

Comparando la Biodanza ${ }^{\circledR}$ con otras técnicas expresivas utilizadas en TMG, la terapia más similar en cuanto a técnica de comunicación y expresión y beneficios obtenidos puede ser Musicoterapia. En la revisión sistemática de Mössler et al. (16) se concluye que la musicoterapia como agregado a la atención habitual ayuda a los pacientes con esquizofrenia a mejorar el estado general, el estado mental (incluidos los síntomas 
negativos) y el funcionamiento social, si se proporciona un número suficiente de sesiones de musicoterapia (más de 20), a cargo de musicoterapeutas expertos. En nuestro caso se han encontrado resultados positivos con 12 sesiones de Biodanza®.

Otras indicaciones de Biodanza ${ }^{\circledR}$ serían en pacientes en los que la mejora de la calidad de vida, la autoestima y el descenso de la ansiedad y depresión sean relevantes, como ocurre en muchas patologías crónicas, como enfermedad mental, fibromialgia, cáncer, diabetes, hipertensión, problemas cardiacos, etc, evitando emplearla mediante un grupo regular en fase aguda, crónica avanzada y/o cuidados paliativos. En estos últimos casos, la metodología se adaptaría para estos pacientes (hospitalizados o no), con músicas y ejercicios personalizados realizados a pie de cama, incluyendo a paciente y familiares. Igualmente, estaría indicada en sanitarios para disminuir el "burnout" y en familiares y cuidadores principales implicados en el proceso de salud.

Las fortalezas de este estudio son: que es el primer trabajo que analiza la efectividad de Biodanza® como complemento al tratamiento estándar en pacientes con TMG y sus familiares en un HD. Estos resultados pueden estar influenciados por la alta adherencia del grupo a la intervención, asistiendo a una media de 9,5 sesiones de 12 totales. Existió una alta motivación en los participantes al inicio de la intervención que se mantuvo durante la misma, desarrollándose un ambiente de confianza, seguridad, escucha, etc., que produjo una buena alianza terapéutica potenciando una mayor adherencia a los otros tratamientos recibidos. Para el éxito de la intervención es necesario realizar una terapia individualizada que tenga en cuenta las necesidades expresadas por cada participante, este es un objetivo fundamental del tratamiento integral en esta población.

Una posible limitación es el pequeño tamaño muestral $(n=17)$ esto es debido entre otros factores al limitado número de pacientes con TMG que se encuentra en seguimiento en los Hospitales de Día con una adecuada adherencia a los tratamientos, aun así futuros estudios con mayor tamaño muestral son necesarios para corroborar los resultados obtenidos. Otra posible limitación es que la investigadora no fue cegada para la evaluación del grupo, pero esta limitación se ha minimizado por el hecho de que el responsable del análisis estadístico no estuvo implicado ni en la recogida de datos a los pacientes ni en el desarrollo del tratamiento. 
Como conclusión, este estudio ha

mostrado que la intervención con Biodanza ${ }^{\circledR}$ junto al tratamiento estándar puede mejorar la calidad de vida y la autoestima y disminuir la ansiedad y depresión en personas con TMG y a sus familiares. Los resultados de esta investigación señalan que la Biodanza ${ }^{\circledR}$ es un apoyo para facilitar a las personas con TMG y sus familias mayor bienestar en su vida. Es necesario proporcionar un número suficiente de sesiones de Biodanza ${ }^{\circledR}$ a cargo de terapeutas expertos con buena alianza terapéutica para provocar cambios significativos. Con los datos obtenidos, no se recomiendan menos de 12 sesiones. Por tanto, para investigaciones futuras sería conveniente realizar estudios en los que se analice en qué medida los efectos de la Biodanza ${ }^{\circledR}$ en personas con TMG y en sus familiares, se mantienen a largo plazo tras la intervención; donde se evalúe las características de la intervención que la hacen más efectiva; el número de sesiones para mantener el efecto; así como un tamaño muestral más elevado y una asignación aleatoria de los participantes y/o un ensayo clínico. 


\section{BIBLIOGRAFÍA}

1. Parabiaghi A, Bonetto $C$, Ruggeri M, Lasalvia A, Leese M. Severe and persistent mental illness: a useful definition for prioritizing community-based mental health service interventions. Soc Psyc Psyc Epid. 2006;41(6):457-63.

2. De la Cámara C, Caro FJ, Escusa M, Galán FJ, Gracia L, Martín A et al. Guía de Práctica Clínica de Intervenciones Psicosociales en el Trastorno Mental grave. Ministerio de Sanidad y Política Social. $1^{\text {a }}$ ed. Madrid: Guía Salud. [1-1-2009]. [1-1-2009; 2-3-2017]. Disponible en: http://portal.guiasalud.es/web/guest/catalogo-gpc

3. Anthony W. Recovery-oriented service systems: Setting some system level standards. Psyc Reh Jour. 2000; 24:159-168.

4. Farkas M, Gagne C, Anthony WA. Recovery and rehabilitation: A paradigm for the new millennium. Boston, MA: Center for Psychiatric Rehabilitation; 1999

5. Harding C, Anthony WA, Chamberlin J, Farkas M. The Recovery Vision: New paradigm, new questions, new answers. World Health Organization Collaborating Center for World Health Day. Boston, MA; 2001

6. Xiong $\mathrm{H}$, Xiong $\mathrm{W}$, Lipeng $\mathrm{F}$, Wang R. Comprehensive family treatment for schizophrenic patients: a prospective, randomized, single blind control trial of 63 patients. Chin Ment Health Jour. 1994;2:5-201.

7. Andreu J, Aznar FJ, Carlson J, Casanovas C, Castells G, Gil J, et al. Guía de Práctica Clínica sobre la Esquizofrenia y el Trastorno psicótico Incipiente. Ministerio de Sanidad y Política Social. $1^{\text {a }}$ ed. Madrid: Guía Salud. [1-3-2009]. [1-1-2009; 4-3-2017]. Disponible en:

http://portal.guiasalud.es/web/guest/catalogo-gpc

8. Kuipers E, Kendall T, Antoniou J, Barnes T, Bhui Kamaldeep, Bird V, et al. Core interventions in the treatment and management of schizophrenia in primary and secondary care (update). Leicester (United Kingdom): National Institute for Health and Clinical Excellence: Guidance; 2009. Disponible en:

http://www.ncbi.nlm.nih.gov/books/NBK11681/

9. Toro Araneda R Biodanza. Tercera edición. Santiago de Chile: editorial índigo/cuarto propio; 2009.

10. Toro Araneda R. International Biocentric Foundation (IBF). www.biodanza.org.

11. Carbonell-Baeza A, Ruiz J.R, Aparicio V.A, Martins-Pereira C.M, Gatto-Cardia M.C, Martínez J.M et al. Multidisciplinary and biodanza intervention for the management of fibromyalgia. Acta Reumatol Port. 2012; 37:240-250.
12. Carbonell-Baeza A, Aparicio V.A, Martins-Pereira C.M, Gatto-Cardia M.C, Ortega F.B, Huertas F.J et al. Efficacy of Biodanza for Treating Women with Fibromyalgia. The Journal of Alternative and Complementary Medicine. 2010;16(11):1191-1200.

13. López-Rodríguez M, Castro-Sánchez A, Fernández-Martínez $M$, Matarán-Peñarrocha $G$, Rodríguez-Ferrer E. Comparación entre biodanza en medio acuático y stretching en la mejora de la calidad de vida y dolor en los pacientes con fibromialgia. At Prim. 2012;44(11):641-649.

14. Segura-Jiménez V., Gatto-Cardia C.M., Martins-Pereira C.M., Delgado-Fernandez M., Aparicio V.A., Carbonell-Baeza A. Biodanza Reduces Acute Pain Severity in Women with Fibromyalgia. Pain Management Nursing. 2017;18(5):318-327. doi: 10.1016/j.pmn.2017.03.007. Epub 2017 Jun 9.

15. López-Rodríguez M.M., Baldrich-Rodríguez I., Ruiz-Muelle A., Cortés-Rodríguez A.E., Lopezosa-Estepa T. \& Roman P. Effects of Biodanza on Stress, Depression, and Sleep Quality in University Students. The Journal of Alternative and Complementary Medicine. 2017;23:556-565. doi: 10.1089/ acm.2016.0365. Epub 2017 Jun 7.

16. Mössler K, Chen X, Heldal TO, Gold C. Music therapy for people with schizophrenia and schizophrenia-like disorders. Cochrane Database of Systematic Reviews. 2011;Issue 12. Art. No.: CD 004025 .

DOI: 10.1002/14651858.CD004025.pub3.

17. Alonso J, Prieto L y Antó JM. La versión española del SF-36 Health Survey (Cuestionario de Salud SF-36): un instrumento para la medida de los resultados clínicos. Med Clin (Barc). 1995;104:771-776

18. Mubarak AR, Barber JG. Emotional expressiveness and the quality of life of patients with schizophrenia. Social Psychiatry and Psychiatric Epidemiology. 2003;7:380-384.

19. Tejero A, Guimerá EM, Farré JM et al. "Uso clínico del HAD (Hospital Anxiety and Depression Scale) en población psiquiátrica: un estudio de su sensibilidad, fiabilidad y validez". Rev Depto Psiquiatría Facultad de Med Barna. 1986;13:233-238.

20. Atienza, L., Moreno, Balaguer, I. An analysis of the dimensionality of the Rosenberg self-esteem scale in a sample of Valencian adolescents. Universitas Tarraconensis. 2000;22:34-45

21. Bobes J, González MP, Bousoño M. Calidad de vida en las esquizofrenias. J.R. Prous. Barcelona. 1995

22. Sederer L, Dickey B. Outcomes assessment in clinical practice. Lippincott Williams and Wilkins. Baltimore. 1996 
23. Lehman AF. A Quality research of Life Interview for the chronically mentally ill. Evaluation and Program Planning. 1988;11(1):51-62.

24. Muñoz M, Pérez, E, López A, Panadero S. Apuntes para la medida de la calidad de vida en la rehabilitación psicosocial de enfermos mentales crónicos. Boletín de la Asociación Madrileña de Rehabilitación Psicosocial. 1999;10(11):20-26.

25. Castañeda GM. La Biodanza como práctica corporal en relación con la promoción de la salud. Educación Física y Deporte. 2009;28(2):81-90.

26. Abad Robles MT, Castillo Viera E, Orizia Pérez AC. Los efectos de un programa motor basado en la biodanza en relación con parámetros de inteligencia emocional en mujeres. Cuadernos de Psicología del Deporte. 2014;14(1):13-22

27. López-Rodríguez M, Castro-Sánchez A, Fernández-Martínez M, Matarán-Peñarrocha $G$, Rodríguez-Ferrer E, Granados Gámez G, et al. Efectividad de la Biodanza ${ }^{\circledR}$ acuática sobre la calidad del sueño, la ansiedad y otros síntomas en pacientes con fibromialgia. Med Clin (Barc). 2013;141:471-8. 